Volume 10, No.6, November - December 2021

International Journal of Advanced Trends in Computer Science and Engineering

Available Online at http://www.warse.org/IJATCSE/static/pdf/file/ijatcse031062021.pdf

https://doi.org/10.30534/ijatcse/2021/031062021

\title{
The Crossbreed Invariant Optimization MSVM Method for Effective Diagnosis of Pneumonia from Chest X-Ray Images
}

\author{
Shivanshu Katoch ${ }^{1}$, Munish Katoch ${ }^{2}$ \\ ${ }^{1}$ Sri Sai University, India, Katochshiva1997@ gmail.com \\ ${ }^{2}$ Sri Sai University, India, munishcse710@ gmail.com
}

Received Date : October 09, 2021 Accepted Date : November 08, 2021 Published Date : December 06, 2021

\begin{abstract}
The survival percentage of pulmonary sufferers can be improved if pneumonia is detected in time. Imaging of the chest $\mathrm{x}$-Ray is the most common way of finding as well as identifying pneumonia. A competent radiologist poses a severe problem while identifying pneumonia using CXR scans. To maximize classification precision, it requires an autonomous computer-aided detection approach. Designing a lightweight autonomous pneumonia detection mechanism for resource-efficient healthcare devices is critical for enhancing healthcare quality while lowering expenses and increasing reaction time. In this proposed work, a machine learning-based hybridization approach is implemented for the identification of pneumonia in the chest x-Ray scans. The proposed methodology is divided into different segments: the 1st segment is to remove noise from the chest $\mathrm{x}$-Ray scans (pre-processing). After the pre-processing of CXR scans, the second module is to extract features from the pre-processed scans. The scale-invariant feature transform (SIFT) method is implemented for the extraction of essential features. This CIO-MSVM (Crossbreed Invariant Optimization-MSVM) method will select the valuable feature with the help of FF (fitness function). This function will help to select the feature matrix and then implement the MSVM algorithm. It will pass the instance selected feature set to the train model and test model. It will classify the feature sets. If feature sets will match then detect or classify the Chest X-ray image and evaluate the performance metrics such as accuracy, spec, sens., etc and compared with the existing methods.
\end{abstract}

Key words : Pneumonia Pulmonary disease, Chest x-Ray, Feature extraction using SIFT, AL-MSVM using Classification.

\section{INTRODUCTION}

Due to environmental change, variability in climate, lifestyle, as well as other reasons, the impact of sickness on wellness is growing substantially. It has elevated the likelihood of illness. Pneumonia is one of the diseases that can occur due to climate variability, environmental change as well as other reasons.
Pneumonia is the most common lungs illness that causes people all over the world. Pneumonia is a devastating illness that affects the lung's air passages to be clogged with mucus or fluid. Pneumonia can be classified into two categories: bacterium and infectious [1]. The consequences of streptococcus pneumonia are much more serious. Every type of infection requires a distinct treatment approach. Antibiotics method is used for treating bacterial pneumonia, whereas the other type of infection normally does not require antibiotic treatment and it will heal by itself. Major pneumonia's cause is the excessive degree of pollution that exists all over the world. Mostly in the United States, it is the eighth leading death cause. In 2017, almost one billion elderly individuals as well as 800,000 children under the age of 5 died with pneumonia, as per the findings [2].

Pneumonia impacts both adults and children all over the globe, although it is more prevalent in sub-Saharan Africa and Southern Asia. For the protection of children against pneumonia, several precautions can be taken, and all these efforts can be executed using basic therapies, low-cost treatments, and low-tech services as well as medications.

The severity of pneumonia can vary from mild illness to life-threatening disease. Toddlers and Infants, individuals over the age of 65 , as well as individuals with chronic difficulties or severe allergies are the most vulnerable [3].

There are several symptoms and signs of pneumonia such as:

- Muscles ache, when cough or breathe

- Alteration in mental ability or disorientation in adults above the age 65 and older

- Phlegm occur in cough

- Fatigue

- Sweating, fever

- Diarrhea, vomiting, nausea

- Breath shortness

Bacterial Pneumonia occurs as a consequence of a respiratory inflammation caused by the fungus, viruses, bacteria, fungus, parasites, and other things. There are different types of bacterial pneumonia given below:

- Community-acquired (CAP): Acute lung tissue inflammation in a person who contracted this in the neighborhood or within 48 hours of hospitalization.

- Hospital-acquired (HAP): After 48 hour's treatment in the hospital, a non-intubated patient experiences a chronic infection of lung parenchyma. 
- Healthcare-Associated (HCAP): A kind of community-acquired pneumonia of the lungs that often occurs 48 hours or more following ventilation system implantation.

- Ventilator-Associated Pneumonia (VAP): Severe pulmonary inflammation obtained from a medical facility including a nursing facility, dialysis facility, intensive outpatient program, or an individual who has been hospitalized in the last 3 months [3].

Pneumonia disease is hard to detect through imaginary scans. There are several challenges occurs in pneumonia detection. Pneumonia is a disease that affects the lungs and is caused by bacteria or fungus. Advanced analysis is an important component of a successful treatment plan. Experts can usually diagnose the condition using X-ray scans of the chest. Various researches have been done in radiograph approaches for the identification of Pneumonia from the chest x-ray images. A chest radiograph is an essential need for the detection of diseases like Tuberculosis and Pneumonia. Surveyed several articles and main problems were found in the detection of pneumonia from chest images. Hence, it becomes necessary to diagnosis this disease at the initial stage. The main problems determined in existing work are time-consuming process, very expensive, activation function, and the pattern recognition problem. This main issue of the activated function is that it has less training function due to fault derivative and smaller saturated region. Hence, the updation of the weight vanishes. On the other hand, there is a presence of an unbalanced sample distribution and insufficient generation of the trained networks. In the presence of a huge database, it becomes difficult to extract the feature points. Pattern recognition may be unmatched, so it becomes confusing to recognize and classify Pneumonia disease. An existing research work used well-defined CNN models like Vgg16 and Xception models for the analysis of Pneumonia disease [4]. This model has utilized transfer learning as well as fine-tuning in the learning phase. The experiment outcomes presented which Vgg-16 network exceeds the Xception model at the accuracy rate of 0.87 percent, 0.82 percent respectively. The research work is implemented a novel model to optimize the existing problems and improve system performance.

As earlier said, pneumonia impacts a massive amount of people, particularly children, primarily in developing and underdeveloped regions severe adverse outcomes like overpopulation, inadequate sanitation, and starvation, as well as a lack of sufficient medical services. Timely screening of pneumonia is essential for successful treatment. The most prevalent method of identification is X-ray imaging evaluation, although it is dependent on the radiologist's interpretation competence and also is often not accepted by the specialists. To detect the condition, an autonomous CAD system with generalizations abilities is necessary. To our recollection, several traditional models for the categorization of infected patients in literature have concentrated on constructing a singular $\mathrm{Cnn}$ architecture, and the use of the hybrid machine learning paradigm in this classification problem has not been investigated. The hybrid machine learning paradigm, on the other hand, combines discriminative features from all of the components training sets, enabling it to generate better recommendations, and was used in this work. To deal with the limited quantity of medical statistics provided, hybrid machine learning paradigm was utilized as classification algorithms.

- In this paper, various pneumonia detection machine learning and deep learning techniques are analyzed.

- A pre-processing process is implemented and feature extraction (FE) by utilizing the scale-invariant feature transform (SIFT) method.

- A hybrid model using the ALO with the MSVM method for diagnosing pneumonia from medical images is implemented in this work.

- The performance metrics like accuracy rate, specificity, precision, recall, and f1-score are used for performance evaluation and several with existing methods are compared with the proposed methodology.

The sections of the papers are as: In first section, a brief introduction about pneumonia with symptoms and signs is explained. The literature of existing techniques of pneumonia detection is surveyed in section 2 . The methods of pneumonia detections are explained in section 3. The proposed methodology is explained in section 4.

\section{LITERATURE REVIEW}

The literature review is crucial in establishing the foundation for the issue and its expected approach. It creates the research group estimates for taken at different time's scenarios. This section surveyed the various existing techniques of PNEUMONIA detection based on chest x-ray images. Singh, S., et al., (2021) [5] studied that convolutional neural networks had demonstrated to be quite effective at detecting objects in images. The authors also found that the enhanced CNN-based technique Quaternion convolutional neural network was a generalization of convolutional layers. Quaternion convolutional neural network combines all different color bands (Red, Green, and Blue) as a cohesive block, extracting more relevant attributes as well as improving categorization. The authors had used publicly released huge Chest x-Ray samples on Kaggle to learn a Quaternion residual network as well as attained 93.75 percent of accuracy of the classification and 0.94 percent of F-score. The authors compared the proposed model with different CNN topologies. The comparison discovered that the Quaternion Residual network had greater classification accuracy. A deep convolutional neural network (DCNN) was presented by Rajasenbagam, et al. (2021) [6] in the proposed methodology to identify Pneumonia disease inside the lungs employing X-ray scans of the chest. A Pneumonia Chest $\mathrm{X}$-ray Database encompassing 12,000 scans of contaminated as well as non-infected chests $\mathrm{X}$-ray scans were used to construct the suggested Deep CNN classifiers. The Chest $\mathrm{X}$-ray8 collection was used for the pre-processing stage as well as creating the database. The visuals mostly in the collection were annotated utilizing Metadata as well as additional information employing the Information features extraction methodology. To boost the collection of images within every category, matrix factorization algorithms were employed. The modified graphics were created using basic 
modification techniques as well as the deep convolutional generative adversarial network. The suggested Deep CNN model was constructed using the VGG19 system. Within unknown lung X-ray scans, the suggested Deep Classification algorithm had an accuracy of classification of 99.34 percent. The suggested deeper CNN's results were compared to those of state-of-the-art transfer learning methods including InceptionNet, AlexNet, and VGG16Net. The comparative findings demonstrate that the suggested Deep CNN model performs better than the other strategies in terms of classification results. Acharya et al., (2020) [7] designed an autonomous detection methodology of pneumonia from images of the chest. To build and evaluate the proposed system for autonomous identification of multiple types of pneumonia infection, the authors were used the Kaggle dataset. The proposed methodology was based on a deep Siamese-based computational framework and the authors analyzed that, there were approaches that rely primarily on the transferable learner or classic customized procedures to categorize the pneumonia condition. To analyze or identify the issue, deep Siamese networks exploit the symmetric structure of the two input images. Every X-ray scan of the lungs was divided into two segments, which were then fed through into the system, and compared the symmetric structure as well as the quantity of illness dispersed over these two regions. The proposed methodology may aid medical practitioners in quickly diagnosing pneumonia using X-ray images. Hashmi, M., et al., (2020) [8] proposed an effective technique for the detection of pneumonia. The main objective of the proposed methodology was to help radiologists for getting to a conclusion. The method used WC (weighted classifier) in which predictions from multiple SOA (state-of-the-art) learning techniques like ResNet18, Xception, InceptionV3, DenseNet121, and MobileNetV3 were used. The outcomes were anticipated based on the standard of the dataset utilized. For tuning of DL (deep learning) schemes, TL (transfer learning) and DA (data augmentation) techniques were used, so that maximum accuracy could be achieved. WC attained 98.4 percent accuracy, 99.7 percent AUC and 99 percent F1 score which proved the effectiveness of the used technique.Ayan, E., et al., (2019) [9] designed a methodology for the detection of pneumonia. The methodology was worked with CXR (chest $\mathrm{X}$-Ray) scans. The deep learning-based technique was used for efficient results. The convolutional neural network (CNN) based pre-trained models were implemented to attain accurate outcomes. The CNN-based VGG16 and Xception CNN models were used in the proposed methodology. The CNN-based VGG16 model had attained better results than the Xception model. VGG16 model had achieved an accuracy of 0.87 percent and the Xception model had attained an accuracy of 0.82 per cent.Varshni et al. (2019) [10] As a result, establishing an autonomous tool for classifying pneumonia might be advantageous for quickly curing the symptoms, especially in remote locations. Convolutional neural networks have attracted a lot of attention for illness categorization due to the popularity of deep learning models in evaluating medical imagery. Furthermore, visual clustering algorithms benefit greatly from selected features using pre-trained Convolutional networks on large amounts of data. The efficiency of pre-trained Convolutional networks used as feature-extractors based on distinct learners for the diagnosis of healthy and diseased $x$-Ray was evaluated in this paper. We use analysis to get the best Classification algorithm for the job. The results of the analysis revealed that using fully convolutional $\mathrm{Cnn}$ architectures and automated classification machine learning to analyze chest X-ray pictures, particularly to diagnose Pneumonia, could be highly advantageous.

The different existing methods for the detection of Pneumonia with issues, performance metrics, and improvements are depicted in Table 1. In Table 2, deep learning-based existing techniques used in Pneumonia identification are compared.

Table 1: Different existing methods of pneumonia detection

\begin{tabular}{|l|l|l|l|l|}
\hline Author/Year & Proposed Method & Problem /Issues & $\begin{array}{l}\text { Performance } \\
\text { Metrics }\end{array}$ & Improvements \\
\hline $\begin{array}{l}\text { Singh, S., et al., } \\
\text { (2021) [5] }\end{array}$ & $\begin{array}{l}\text { Deep } \\
\text { learning-based } \\
\text { Quaternion } \\
\text { convolutional } \\
\text { neural network }\end{array}$ & $\begin{array}{l}\text { Need to extract } \\
\text { more essential } \\
\text { features for more } \\
\text { accurate results of } \\
\text { classification }\end{array}$ & $\begin{array}{l}\text { Accuracy } \\
\text { F1-score } \\
\text { Recall } \\
\text { Precision }\end{array}$ & $\begin{array}{l}\text { With more layers as } \\
\text { well as features, the } \\
\text { suggested Deep } \\
\text { Convolutional } \\
\text { neural model can be } \\
\text { enhanced. It'll also } \\
\text { allow healthcare } \\
\text { providers to } \\
\text { diagnose pulmonary } \\
\text { disorders from lung } \\
\text { X-ray scans at an } \\
\text { initial phase of the } \\
\text { infection with a low } \\
\text { prevalence rate. }\end{array}$ \\
\hline $\begin{array}{l}\text { Rajasenbagam, et } \\
\text { al. (2021) [6] }\end{array}$ & $\begin{array}{l}\text { Deep CNN with } \\
\text { pre-trained } \\
\text { classifier model }\end{array}$ & $\begin{array}{l}\text { At the initial stages } \\
\text { of the illness, low } \\
\text { prevalence }\end{array}$ & $\begin{array}{l}\text { Recall } \\
\text { Accuracy } \\
\text { Precision }\end{array}$ & $\begin{array}{l}\text { For better results, } \\
\text { CNN layers will be } \\
\text { upgraded. }\end{array}$ \\
\hline Acharya et al., & Deep & Inefficient results on & AUC Score & Hybrid techniques \\
\hline
\end{tabular}




\begin{tabular}{|c|c|c|c|c|}
\hline (2020) [7] & $\begin{array}{l}\text { learning-based } \\
\text { framework }\end{array}$ & challenging frames & & $\begin{array}{l}\text { will be implemented } \\
\text { to attain the efficient } \\
\text { results }\end{array}$ \\
\hline $\begin{array}{l}\text { Hashmi M. F., et } \\
\text { al., (2020) [8] }\end{array}$ & $\begin{array}{l}\mathrm{CNN} \\
\text { (convolutional } \\
\text { neural network) } \\
\text { architectures, TL } \\
\text { (transfer learning), } \\
\text { Pre-trained NN } \\
\text { (neural network) }\end{array}$ & $\begin{array}{l}\text { The difficulty with } \\
\text { this condition is that } \\
\text { the traits that } \\
\text { describe the } \\
\text { condition's basic } \\
\text { presence can } \\
\text { sometimes be } \\
\text { confused with those } \\
\text { of other illnesses, } \\
\text { thus X-ray pictures } \\
\text { are used to identify } \\
\text { it. }\end{array}$ & $\begin{array}{l}\text { ROC (receiver } \\
\text { operating } \\
\text { characteristics) } \\
\text { Accuracy } \\
\text { Precision } \\
\text { Recall } \\
\text { F1-score } \\
\text { AUC (area under } \\
\text { the curve) }\end{array}$ & $\begin{array}{l}\text { It might be } \\
\text { interesting to } \\
\text { observe approaches } \\
\text { for estimating the } \\
\text { outstanding } \\
\text { outcome matching } \\
\text { to multiple models } \\
\text { more efficiently, as } \\
\text { well as a framework } \\
\text { that incorporates the } \\
\text { diagnosis and } \\
\text { treatment plan while } \\
\text { making } \\
\text { categorization. }\end{array}$ \\
\hline $\begin{array}{l}\text { Ayan, E., et al., } \\
\text { (2019) [9] }\end{array}$ & $\begin{array}{l}\text { Transfer learning } \\
\text { based methodology }\end{array}$ & Inefficient outcomes & $\begin{array}{l}\text { Accuracy } \\
\text { Precision } \\
\text { Specificity } \\
\text { Recall } \\
\text { Sensitivity } \\
\text { F1-score }\end{array}$ & $\begin{array}{l}\text { Multiple networks } \\
\text { will merge with the } \\
\text { proposed work. The } \\
\text { framework will be } \\
\text { able to integrate the } \\
\text { capabilities of two } \\
\text { or more networks } \\
\text { and produce better } \\
\text { outcomes when } \\
\text { detecting } \\
\text { pneumonia using } \\
\text { chest x-Ray scans } \\
\text { this way. }\end{array}$ \\
\hline $\begin{array}{l}\text { Varshni et al. } \\
(2019) \text { [10] }\end{array}$ & $\begin{array}{ll}\mathrm{CNN} & \text { based } \\
\text { framework } & \end{array}$ & $\begin{array}{l}\text { High computational } \\
\text { time }\end{array}$ & AUC Score & $\begin{array}{l}\text { The proposed model } \\
\text { will be enhanced to } \\
\text { attain less } \\
\text { computational time }\end{array}$ \\
\hline
\end{tabular}

Table 2: Comparison of existing deep learning-based techniques of pneumonia detection

\begin{tabular}{|c|c|c|c|}
\hline Author/Year & $\begin{array}{l}\text { Compared } \\
\text { techniques }\end{array}$ & Dataset & Results \\
\hline $\begin{array}{l}\text { Singh, S., et } \\
\text { al., (2021) [5] }\end{array}$ & $\begin{array}{l}\text { Transfer learning } \\
\text { VGG16-Net } \\
\text { Inception-Net } \\
\text { Alex-Net }\end{array}$ & Chest x-ray8 & $\begin{array}{l}\text { Accuracy }=99.3 \% \\
\text { F1 score }=99.29 \% \\
\text { Recall }=98.6 \% \\
\text { Precision }=100 \%\end{array}$ \\
\hline $\begin{array}{l}\text { Rajasenbagam, } \\
\text { et al. (2021) } \\
{[6]}\end{array}$ & $\begin{array}{lr}\text { VGG-16 } & \text { Net, } \\
\text { AlexNet, Inception } \\
\text { V3 Net }\end{array}$ & Chest $\mathrm{x}$-ray8 dataset & $\begin{array}{l}\text { Accuracy } 99.34 \% \\
\text { Recall } 98.6 \\
\text { Precision } 100\end{array}$ \\
\hline $\begin{array}{l}\text { Acharya et al., } \\
(2020)[7]\end{array}$ & - & Kaggle dataset & AUC Score $82.2 \%$ \\
\hline $\begin{array}{l}\text { Hashmi M. F., } \\
\text { et al., (2020) } \\
{[8]}\end{array}$ & $\begin{array}{l}\text { InceptionV3, } \\
\text { ResNet18, } \\
\text { MobileNetV, } \\
\text { Xception, and } \\
\text { DenseNet121 }\end{array}$ & $\begin{array}{l}\text { Pneumonia dataset Guangzhou } \\
\text { Women and Children's Medical } \\
\text { Center }\end{array}$ & $\begin{array}{l}\text { Accuracy } 98.43 \% \\
\text { Precision } 98.26 \% \\
\text { Recall } 99 \% \\
\text { AUC Score } 99.76 \%\end{array}$ \\
\hline $\begin{array}{l}\text { Ayan, E., et al., } \\
(2019)[9]\end{array}$ & $\begin{array}{l}\text { Xception } \\
\text { VGG16 }\end{array}$ & $\begin{array}{l}\text { Collection of } 5856 \text { scans of chest } \\
\text { x-Ray }\end{array}$ & $\begin{array}{l}\text { Accuracy }=0.87 \\
\text { Precision }=0.91 \\
\text { Specificity }=0.91 \\
\text { Recall }=0.89 \\
\text { Sensitivity }=0.82 \\
\text { F1-score }=0.90\end{array}$ \\
\hline $\begin{array}{l}\text { Varshni et al. } \\
(2019) \text { [10] }\end{array}$ & $\begin{array}{l}\text { XCeption, VGG-19, } \\
\text { ResNet-50 }\end{array}$ & Chest radio-graphs dataset & AUC Score $80.02 \%$ \\
\hline
\end{tabular}




\section{PROPOSED DISEASE DETECTION METHODS WITH CHEST X-RAY IMAGES}

The pneumonia detection process is divided into different parts: the first part is the pre-processing part in which the filtration process is implemented. After that, processed image is implemented for the next part (feature extraction). In the second part, features are extracted from the image. The essential features are selected in the feature selection phase. At last, the classification process is applied to the processed image and categorized into different categories. There are various methods are used in the pneumonia detection approach. Methods with Pseudo code are explained below as:

\subsection{Feature Extraction}

SIFT generates a set of descriptors from a visual using a scale-invariant feature transform. Imagery translations, transformation, as well as magnification have little effect on the retrieved descriptor (zoom-out). SIFT descriptors have also been shown to be resistant to a variety range of vision alterations, including modest variations in orientation, distortion, distortion, luminance shifts, and image distortion while maintaining discriminatory enough to be used for comparison [11].

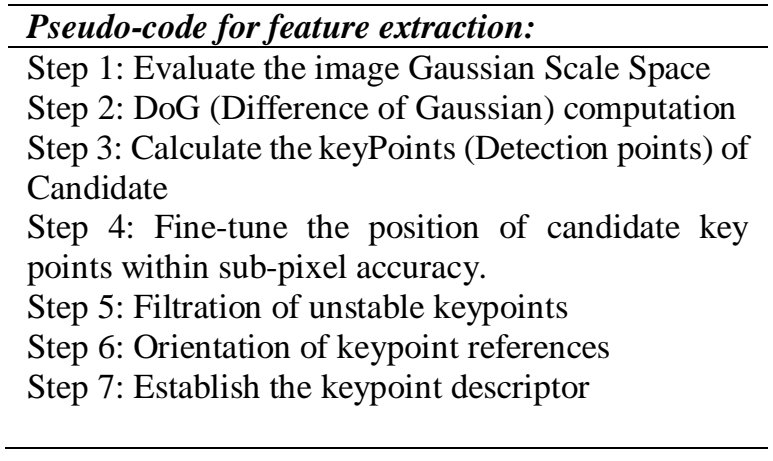

\subsection{Classification by using CIO-MSVM Model}

The Ant lion optimization approach can handle the variable selection issues, and it is quite easy for the ALO approach to get through the localized optimum solution. The Multi-Support vector machine method is used to increase the Ant lion optimization computation globally finding effectiveness. The features of the PNEUMONIA virus are similar to the other diseases. The identification of the PNEUMONIA virus in $\mathrm{x}$-ray images is a challenging task.

Ant lion optimization is the algorithmic framework that mathematically is a blueprint of the interaction between antlions and ants in nature. Antlions are known as doodlebugs sometimes. The life of the ANTLION is divided into two phases where the first is larvae, and the second one is the adult phase. The lifespan of the adult phase is three to five weeks. Their hunting action is fascinating when they are in phase one (Larvae phase). Antlions make the small traps in the shape of a cone, which can be observed in nature, to capture ants. Antlions lay beneath that trap and hold back for prey to be trapped. After chowing down on the prey's flesh, the leftovers are tossed outside the pit by antlions, following which that pit is amended for the next hunt. It has been noticed that bigger pits are dug up by antlions when they are hungry, and this is exactly the main insight for this algorithm [12].

\section{Pseudo-code for feature extraction method: \\ INPUT: ANTS and ANTLIONS, ITERATION \\ OUTPUT: Elitist ant lion and their fitness value (FV) \\ Step 1: Initialization of the agents (random locations) inside the upper bound (UB) and lower bound (lb). \\ Step 2: Evaluate the population fitness value. \\ Step 3: Elite antlion selection. \\ While (until the condition is not met) \\ do \\ for (every ANT) do \\ Step 4: Selection the antlion A with the roulette wheel \\ Step 5: Run ANT random walk corresponding to ANTLION}

Step 6: ANT location updation

Step 7: Fitness value updation

Step 8: Generate the fitness metric and select the

fittest agent

Step 9: Elite ant updation (if new Antlion is better)

Various existing machine learning approaches are used to detect features of the PNEUMONIAvirus. But the methods have low accuracy and more false result detection. In this proposed work, a machine learning-based approach is implemented for the identification of the PNEUMONIA virus. The proposed approach is based on a machine learning-based hybrid classification model. The Multi-support vector machine is implemented for classification purposes. It is used to classify more than two classes. PNEUMONIA has several features and it is hard to select essential features. In the proposed approach Ant Lion optimization is used to select essential features from the extracted features. The hybridization of ALO and MSVM is implemented for efficient results. The chest x-ray image samples are collected from the dataset and then pre-processing is performed on image samples. The unwanted noise is removed in pre-processing phase. The processed image is implemented for the next phase in which features are extracted from the images [13]. The most important step of any process is feature selection. Selecting the essential features from the extracted features is a very crucial task. For this process, the Ant Lion optimization approach is implemented. Then the selected features are further processed for classification purposes. The Multi-Support Vector Machine is implemented for the categorization of features. In the end, the PNEUMONIA virus is detected with the image samples.

\footnotetext{
Pseudo-code for CIO-MSVM Model

Step 1: Candidate_MSupport Vector (MSV) =

\{Select the closet pair from opposite classes\}

Step 2: while (violating point)

do \{ find violator Candidate_MSupport Vector
} 
$($ MSV) $=$ Candidate_MSupport Vector (MSV) U

Violator If $\alpha p<0$

then Candidate_MSupport Vector (MSV) = Candidate $(\mathrm{SV}) / \mathrm{p}$ repeat till all such points are pruned end if end while

\section{\}}

\section{PROPOSED METHODOLOGY}

In an initial phase, search the dataset from the search engine via Google. This engine provides several dataset links. It will prefer the KAGGLE and UCI Dataset repository sites. They both are giving the chest X-ray image in the form of NORMAL and ABNORMAL. The research work will divide the two modules like Training and Testing module. The steps of the proposed methodology are depicted in figure 1.A training and testing module means to design the knowledge domain for understanding the computer or machine. Firstly, upload the dataset Chest X-ray images from the train folder (Multiple Image). After that, the uploaded image will interchange the resize image into the 0-255 range. It will convert the three-dimensional to two-dimensional image i.e., grayscale format image. After this process will add the artificial noise to check the unwanted noise is present or not. If unwanted noises will present then will apply the filtration method to calculate the smooth image. Then, the region of interest values will calculate and develop the FE (feature extraction) method using SIFT approach.

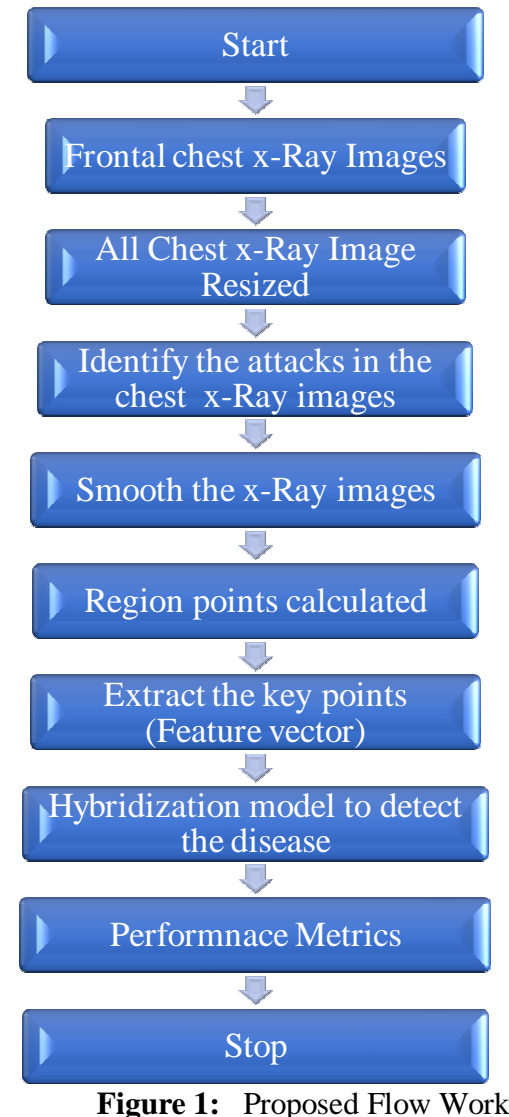

Figure 1: Proposed Flow Work

This approach will extract the feature KPs (key points) in the chest X-ray images. This method will extract the unique properties and then implement the hybrid optimization algorithm using ALO with MSVM.This crossbreed invariant optimization-MSVM method will select the valuable feature with the help of FF (fitness function). This function will help to select the feature matrix and then implement the MSVM algorithm. It will pass the instance-selected feature set to the trained model and test model. It will classify the feature sets. If feature sets will match then detect or classify the Chest $\mathrm{X}$-ray image and evaluate the performance metrics such as accuracy, specificity, sensitivity, etc., and compare with the existing methods.

\section{RESULT AND DISCUSSIONS}

\subsection{Dataset}

In this database [14], upgraded rendition (with 6 more illness classifications and more pictures too) of the dataset utilized in the on-going work which is around multiple times the quantity of frontal chest $\mathrm{x}$-beam pictures. Our dataset is extricated from the clinical PACS database at the National Organizations of Wellbeing Clinical Centre and comprises $\sim 60 \%$ of all frontal chest $\mathrm{x}$-beams in the medical clinic. Subsequently, we expect this dataset is altogether increasingly agent to the genuine patient populace disseminations and reasonable clinical finding difficulties than any past chest $\mathrm{x}$-beam datasets. The size of our dataset, as far as the all-out quantities of pictures and thorax infection frequencies, would better encourage profound neural system preparing.

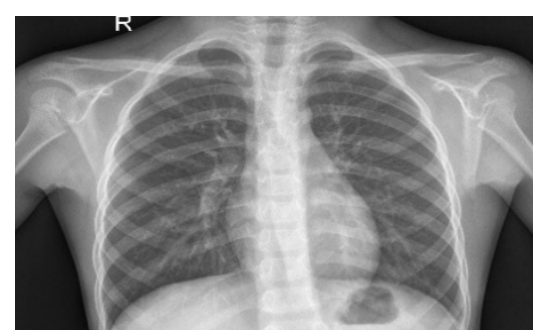

(i)

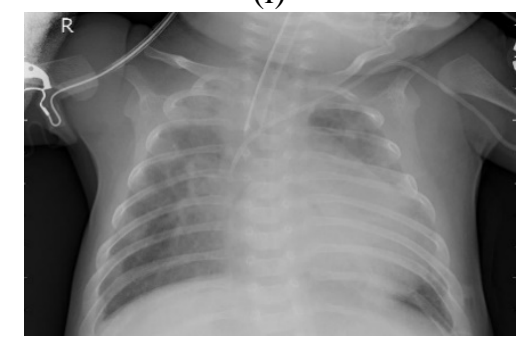

(ii)

Figure 2: (i) Normal and (ii) Pneumonia X-ray Images

\subsection{Performance Metrics}

In this section, different performance parameters are elaborated that is used for the examination of the proposed methodology performance. Accuracy, specificity, sensitivity, precision, recall, f-score, and execution time are all performance metrics used for the evaluation of the methodology. The reliability of the methodology is dependent upon the outcome values of the metrics. 


$$
\begin{aligned}
& \text { Specificity }=\frac{T N}{T N+F P} \\
& \text { Sensitivity }=\frac{T P}{T P+F N} \\
& \text { Precision }=\frac{T P}{T P+F P} \\
& \text { Recall }=\frac{T P}{T P+F N} \\
& \text { F-Sore }=2 * \frac{\text { Recall } * \text { Precision }}{\text { Recall }+ \text { Precision }}
\end{aligned}
$$

\subsection{Simulation Tool}

MATLAB is a vector simulator that was created to make software developed using the simplified mathematical package and Eigen system package (EISPACK) methods as simple as possible. MATLAB is the abbreviation of Matrix Laboratory. MATLAB is a sophisticated development framework with enhanced data types, constructed scripting and testing facilities, and OOPs (object-oriented programming) capabilities. MathWorks created the MATLAB coding language. It began as a basic algebraic equations software package and a matrix scripting language. MATLAB is a high-level programming language for improving practical calculation.

\subsection{Results}

The proposed pattern is developed in the matrix laboratory simulation tool with GUIDE has developed a PROJECT-BASED DESKTOP APPLICATION. In the result and discussion, two categories of chest X-ray images were kept under DIC (digital image camera) and almost 200 samples from each Chest X-ray images were covered in "TRAINING" module. The "TESTING" module has evaluated the proposed novel classification model, continuing data set image of CHEST X-RAY images were chosen for the testing module. Designed a simulation analysis of proposed work, Chest X-ray image (*.jpeg) were saved in the defined image format. The CAD system for infected image detect chest X-ray images into two categories (i) Normal and (ii) Phenomena images are normally based on features (region, and contrast etc). The research work depends on feature vectors and various metrics are evaluated the performance in MIP (medical image processing) approaches like edges and filtration etc. This proposed work has enhanced the arithmetic formulas and compared with the existing techniques.

The research arithmetic formulas are defined in below:
1. Accuracy Rate
2. SP (Specificity Rate)
3. SN (Sensitivity rate), and
4. Precision
5. Recall
6. Time
7. F-Score

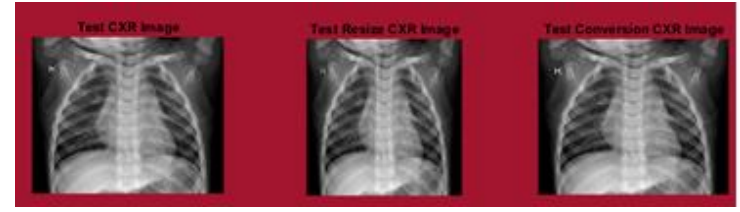

Figure 3: Input Image Acquisition

Fig 3 shows the upload of the test image forms the test folder. It converts the input image $3 \mathrm{D}$ into $2 \mathrm{D}$ dimension image is called GSI (gray scale image). It resizes the uploaded image into 0-255 range.

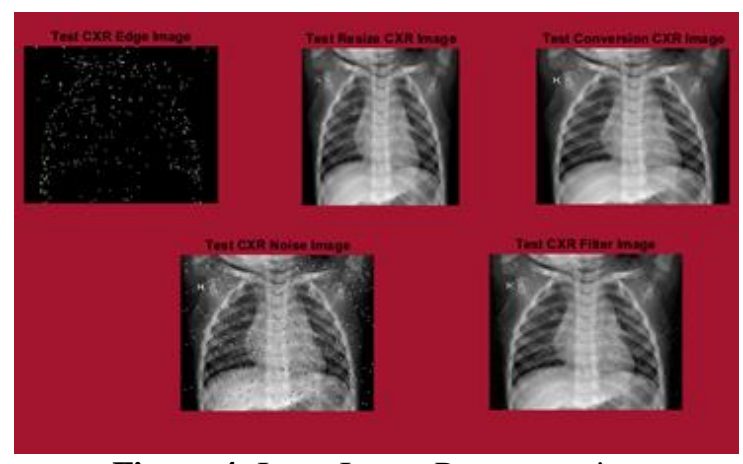

Figure 4: Input Image Pre-processing

Fig 4 shows filtered the uploaded test image with the help of "Filter" method. This Filter method is used in MIP (medical image processing) to enhance the distortion stage in the converted image. It attains improved the image by effectively distribution out the most reliable values i.e, widening out the IR (intensity range) of the x-ray image.

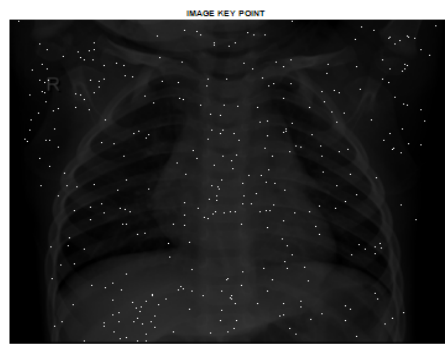

Figure 5: Feature Extraction

Fig 5. shows the SIFT utilizes a KPs (Key Points) to project database into an HD (high-dimensional) FS (feature space), where it is LS (linearly separable). It is same as the idea of SVMs. It extracts the unique quantity feature sets in the form of matrix format.

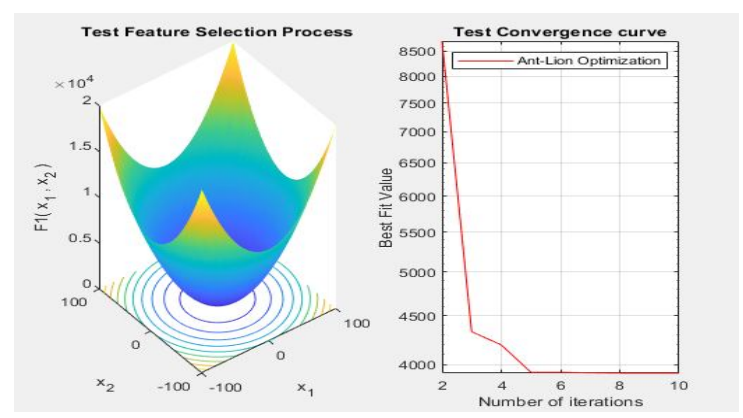

(i) 


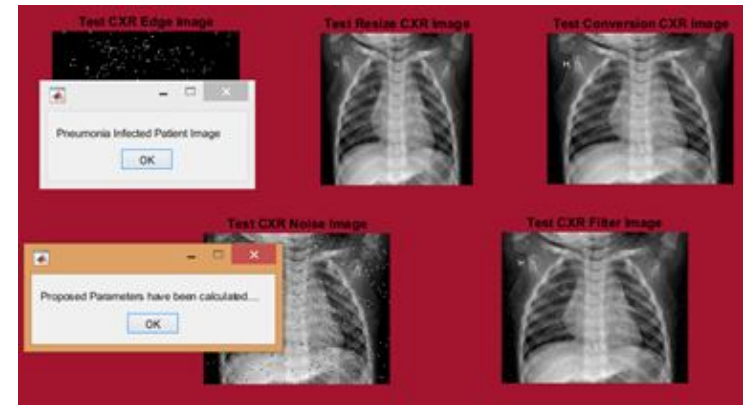

(ii)

Figure 6: Classification using CIO-MSVM method

Fig 6(i) shows the IS (instance selection) three-dimensional graph and test convergence curve in line plot graph used. It calculates the selected FV (feature vectors) with the help of best cost fitness function. This fitness function shows the best outfit selected feature vector which can process for the classification module. Fig 6 (ii) defines the detection process using Crossbreed Invariant Optimization MSVM method. This method has used to detect the disease in the chest X-ray images. This approach has got a high-performance rate and minimum error rates.

Fig 7,8,9,10,11,12, and 13 show the six types of graph plotting with a comparison between proposed and existing parameters such as Acc rate (\%), SP (\%), SN (\%) Pre (\%), Rec(\%), Time (msec), F-Score in per cent, and reduced time consumption. The proposed approach has improved the accuracy rate and time consumption compared with different types of approaches like CIO-MSVM classification method, Vgg16, and Xception classifiers.

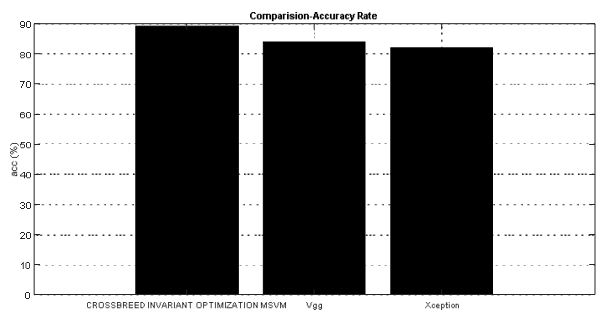

Figure 7: Comparison with proposed using CIO-MSVM method and existing methods: Accuracy Rate

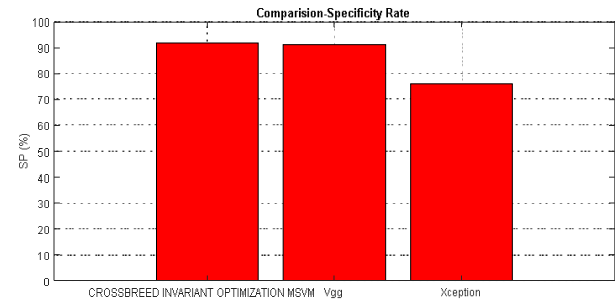

Figure 8: Comparison with proposed using CIO-MSVM method and existing methods: SP (Specificity)

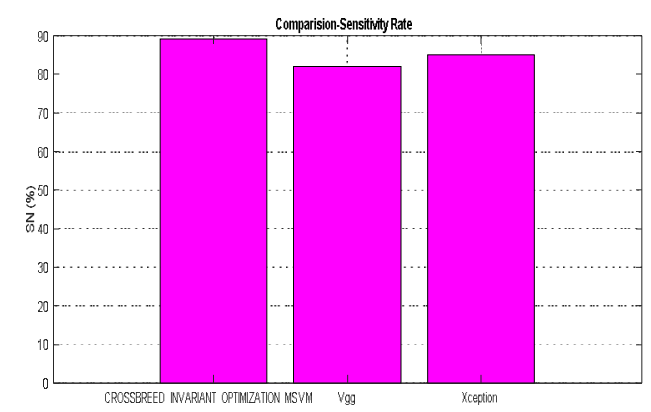

Figure 9: Comparison with proposed using CIO-MSVM method and existing methods: SN (Sensitivity)

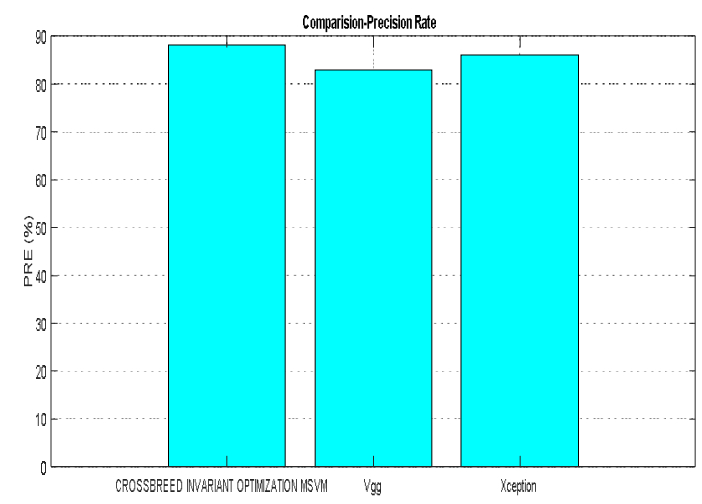

Figure 10: Comparison with proposed using CIO-MSVM method and existing methods: Precision

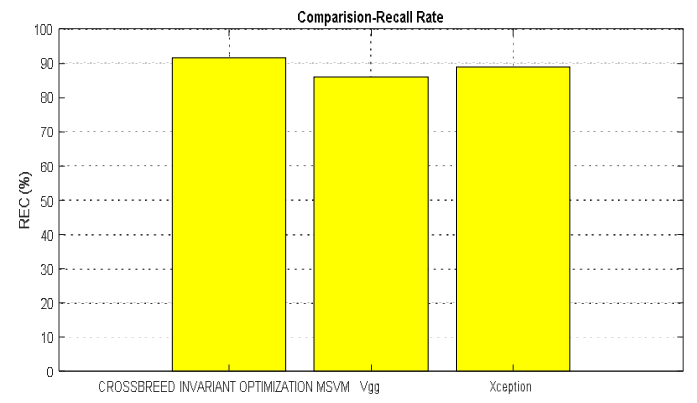

Figure 11: Comparison with proposed using CIO-MSVM method and Existing methods: Recall

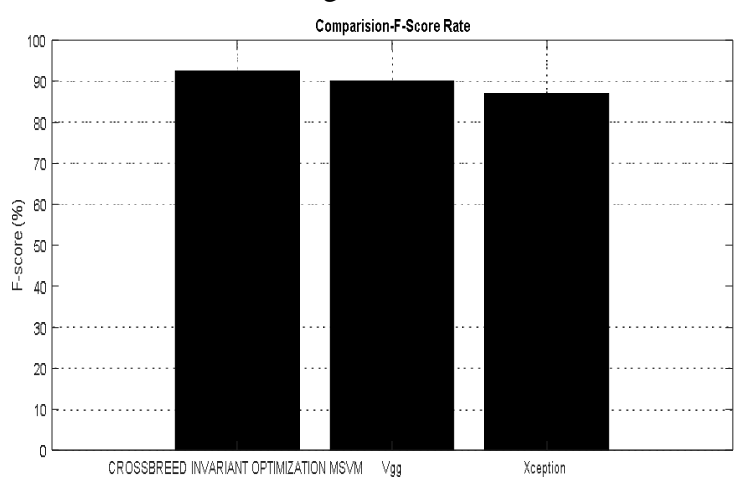

Figure 12: Comparison with proposed using CIO-MSVM method and existing methods: F-Score 


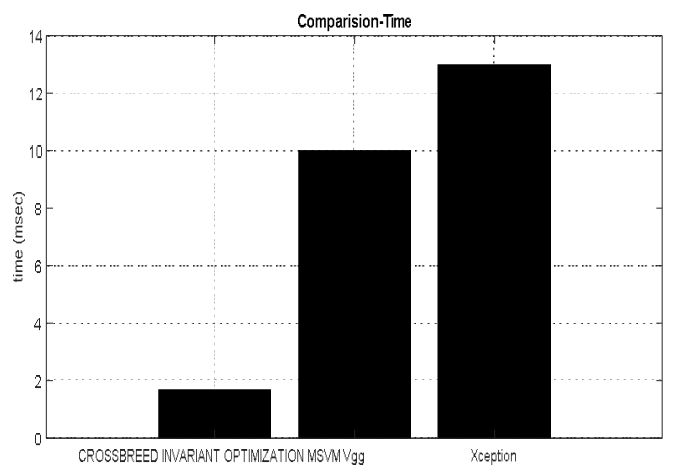

Figure 13: Comparison with proposed using CIO-MSVM method and existing methods: Time

Table 3. Comparison Analysis

\begin{tabular}{|c|l|c|c|}
\hline Parameters & CIO-MSVM & Vgg16 & Xception \\
\hline $\begin{array}{c}\text { Accuracy Rate } \\
(\%)\end{array}$ & $89.10 \%$ & 84 & 82 \\
\hline SP $(\%)$ & $91.68 \%$ & 91 & 76 \\
\hline SN (\%) & $0.89 \sim 89 \%$ & 82 & 85 \\
\hline Precision (\%) & $0.88 \sim 88 \%$ & 83 & 86 \\
\hline Recall (\%) & $91.58 \%$ & 86 & 89 \\
\hline F-Score (\%) & $92.47 \%$ & 90 & 87 \\
\hline Time (msec) & $\begin{array}{l}16.90 \\
\text { millisecond }\end{array}$ & $10 \mathrm{sec}$ & $13 \mathrm{sec}$ \\
\hline
\end{tabular}

Above table 3 shows the existing system performance with existing approaches using Vgg16 and Xception deep learning algorithm accuracy rate is $89.10 \%, \operatorname{Vgg} 16$ is $84 \%$ and Xception classifier accuracy performance is 82 per cent. The existing Vgg16 approach SP performance value is $91 \%$, Xception is 76\%, CIO-MSVM value is $91.68 \%$. The existing Vgg16 approach SN performance value is $82 \%$, Xception is $85 \%$ and proposed CIO-MSVM classifier value is $89 \%$.

\section{CONCLUSION AND FUTURE SCOPE}

In this work, crossbreed invariant optimization -MSVM approach is implemented for the detection of pneumonia disease. There are several symptoms and signs of pneumonia such as fatigue, diarrhoea, vomiting, breathing shortness, muscles ache, etc. Bacterial pneumonia has various types: Community-acquired (CAP), Healthcare-Associated (HCAP), and Ventilator-Associated Pneumonia (VAP). Various challenges occur during pneumonia detection such as low accuracy, high computation, etc. To reduce the previously mentioned challenges, the crossbreed approach is implemented. The crossbreed approach is based on the optimization machine learning approach. The Ant lion and MSVM approach are implemented for efficient results of classification. The experiment analysis of research CIO-MSVM is also compared with other DL classification methods like Vgg16, and Xception. These deep learning networks have minimum generalization abilities and precision compared to the research system. The attained outcomes have determined the high accuracy rate of the research CIO-MSVM. The proposed model has attained the high accuracy rate 89.10 per cent, Precision value of 0.88 , Recall value of 91.5, and Time rate value of 19.92 milliseconds. The proposed CIO-MSVM model has achieved the performance metrics and compared with existing DL methods.

The deep learning-based approach has various advantages in the medical field. In the future, for more efficient results, a deep learning-based approach will be implemented. Also, deep learning-based pre-trained models extracted features more efficiently than the handcrafted feature extraction process. In the future, pre-trained models will use for effective outcomes.

\section{REFERENCES}

1. Chandra, T. B., and Verma, K. Pneumonia detection on chest $x$-ray using machine learning paradigm, In Proceedings of $3 \mathrm{rd}$ international conference on computer vision and image processing, pp. 21-33. Springer, Singapore, 2020.

2. Varshni, D., Thakral, K., Agarwal, L., Nijhawan, R., and Mittal, A. Pneumonia detection using CNN based feature extraction, In 2019 IEEE international conference on electrical, computer and communication technologies (ICECCT), pp. 1-7. IEEE, 2019.

3. Sattar, S. B. A., and Sharma, S. Bacterial pneumonia,StatPearls Publishing, Treasure Island (FL), 19 Jul 2018.

4. Gabruseva, T., Poplavskiy, D., and Kalinin, A. Deep learning for automatic pneumonia detection., Proceedings of the IEEE/CVF Conference on Computer Vision and Pattern Recognition Workshops. 2020.

5. Singh, S., and Tripathi, B. K.Pneumonia classification using quaternion deep learning., Multimedia Tools and Applications 1-22, 2021.

6. Rajasenbagam, T., Jeyanthi, S., and Pandian, J. A. Detection of pneumonia infection in lungs from chest $X$-ray images using deep convolutional neural network and content-based image retrieval techniques. Journal of Ambient Intelligence and Humanized Computing, 1-8, 2021.

7. Acharya, A. K., and Satapathy, R. A deep learning based approach towards the automatic diagnosis of pneumonia from chest radio-graphs. Biomedical and Pharmacology Journal 13.1, pp. 449-455, 2020

8. Hashmi, M. F., Katiyar, S., Keskar, A. G., Bokde, N. D., \& Geem, Z. W. Efficient pneumonia detection in chest xray images using deep transfer learning.Diagnostics 10.6 pp. 417, 2020

9. Ayan, E., and Ünver, H. M. Diagnosis of pneumonia from chest X-ray images using deep learning., 2019 Scientific Meeting on Electrical-Electronics \& Biomedical Engineering and Computer Science (EBBT). IEEE, 2019 April.

10. Varshni, D., Thakral, K., Agarwal, L., Nijhawan, R., and Mittal, A.Pneumonia detection using CNN based feature extraction.2019 IEEE international conference on electrical, computer and communication technologies (ICECCT). IEEE, 2019.

11. Lindeberg, Tony. Scale invariant feature transform. (2012): 10491. 
12. awbaa, H. M., Emary, E., and Parv, B. Feature selection based on antlion optimization algorithm. In 2015 Third world conference on complex systems (WCCS) (pp. 1-7). IEEE. 2015

13. Elkorany, A. S., and Elsharkawy, Z. F. COVIDetection-Net: $\quad$ A tailored PNEUMONIAdetection from chest radiography images using deep learning, Optik, 231, pp. 166405 2021.

14. Rui P, Kang K. National Ambulatory Medical Care Survey: Emergency Department Summary Tables. Table 27". Available from: www.cdc.gov/nchs/data/nhamcs/webtables/2015e dwebtables.pdf, 2015. 